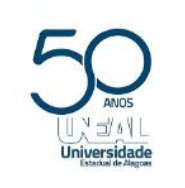

Revista Ambientale

Revista da Universidade Estadual de Alagoas/UNEAL

e-ISSN 2318-454X, Ano 12, Vol. 12 (4), outubro/dezembro (2020)

\title{
Capture of the radical 2,2-diphenyl-1-picryl-hydrazil by Maytenus rigida Mart 2,2-diphenyl-1-picryl-hydrazyl capture by Maytenus rigida Mart
}

\section{Captura do radical 2,2-difenil-1-picril-hidrazila pela Maytenus rigida Mart 2,2-diphenyl-1-picryl-hydrazyl capture by Maytenus rigida Mart}

\author{
Hanna Barbara Araujo QUINTINO (1); Mariana Tenório de Holanda CAVALCANTE ${ }^{(2)}$; Julielle dos \\ Santos MARTINS ${ }^{(3)}$; Maria Lúcia Viera de Britto PAULINO ${ }^{(4)}$; Jonathan Augusto da SILVA ${ }^{(5)}$; \\ Aldenir Feitosa dos SANTOS ${ }^{(6)}$ \\ (1) Estudante de Medicina do Centro Univesitário CESMAC, Campus Maceió \\ *E-mail: hannabaquintino@gmail.com \\ (2) Estudante de Medicina do Centro Universitário CESMAC, Campus Maceió \\ * E-mail: mari_tenorio_95@ hotmail.com \\ (3) Estudante de Medicina do Centro Universitário CESMAC, Campus Maceió \\ *E-mail: juliellemartins@gmail.com; \\ (4) Estudante de Medicina do Centro Universitário CESMAC, Campus Maceió \\ *E-mail:brittomalu@ hotmail.com; \\ (5) Estudante de Química do Instituto Federal de Alagoas \\ * E-mail: jonathan23augusto@gmail.com; \\ (6) Professora do Centro Universitário CESMAC e da Universidade Estadual de Alagoas (UNEAL) \\ *E-mail:aldenirfeitosa@gmail.com. \\ *E-mail do autor correspondente: E-mail: hannabaquintino@gmail.com
}

Recebido em outubro e aceito em dezembro 2020.

Resumo - A espécie Maytenus rigida Mart, conhecida por "Bom-homem", "Bom-nome", "Cabelo-de-negro", "Casca-grossa" e "Pau-de-colher", é uma arvore de pequeno porte que faz parte da família Celastracea e porta aspectos terapêuticos, sendo empregada em casos de dores em especial contra afecções gástricas, renais, uterinas e dermatológicas. O estudo tem o objetivo de avaliar o potencial de captura do radical 2,2-DIFENIL-1-PICRIL-HIDRAZILA pela da casca da $M$. rígida, associado com estudos laboratoriais, como prospecção fitoquímica. A atividade antioxidante foi determinada através do método DPPH (2,2-difenil-1-picril-hidrazil). Já a prospecção fitoquímica seguiu os parâmetros determinados por Matos (1989). A avaliação da captura do radical orgânico pelo extrato etanólico da casca da Maytenus rigida mart mostrou que a espécie apresenta atividade anti-radicalar, o que permite inferir que realmente tem dentre seus componentes metabólitos secundários da classe dos antioxidantes, como compostos fenólicos, flavonoides, catequinas e outros. A prospecção fitoquímica, por sua vez, foi capaz de demostrar mais especificamente quais classes de substâncias antioxidantes estavam presentes no extrato, sendo elas: flavonoides, xantonas, chalconas, auronas, flavononois, catequinas, flavononas, xantonas, esteroides livres e saponinas. Pode-se considerar que a espécie $M$. rigida apresenta capacidade redutora, podendo atuar no combate de doenças, sinais e sintomas que tem base fisiopatológica no estresse oxidativo, como é o caso de doenças inflamatórias e neoplásicas, dando, dessa forma, respaldo científico a aplicação empírica do vegetal pelo conhecimento popular.

Palavras-chave: Atividade anti-radicalar. Bonome. Metabólitos secundários. 


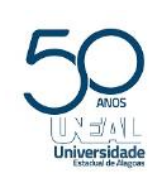

Revista Ambientale

Revista da Universidade Estadual de Alagoas/UNEAL e-ISSN 2318-454X, Ano 12, Vol. 12 (4), outubro/dezembro (2020)

\begin{abstract}
The specie Maytenus rigida Mart, known as "Bom-homem", "Bom-nome", "Cabelode-negro"; "Casca-grossa" e "Pau-de-colher", is a small size tree that belongs to the Celastracea Family and has therapeutical aspects, being used to treat pain, mostly in digestive, urinary, uterine and skin conditions. This article aims to evaluate $M$. rígida Mart peel's capacity to capture 2,2-dyphenyl-pycril-hydrazil radical, associated to laboratory studies such as phytochemical prospecting. The evaluation of $M$. rigida ethanolic extract capacity to capture the organic radical (DPPH) showed that this species has antirradical activity, wich allows to conclude that among its compounds there may be secondary metabolites carring antioxidante activity, such as phenolic compounds, flavonoids and catechins. It may be considered that $M$. rigida has reductive capacity, and so, it may be used to fight diseases and comum symptoms wich originate in oxidative distress, as it happens in inflamatory e neoplastics diseases, creating in this matter, cientific grounds to the experimental use of this vegetable by brazilian population.
\end{abstract}

Keywords: Antiradical activity. Bonome. Secondary metabolites.

\title{
Introdução
}

A espécie Maytenus rigida Mart (Celastraceae), conhecida por "Bom-homem", "Bomnome", "Cabelo-de-negro", "Casca-grossa" e "Pau-de-colher", é uma arvore de pequeno porte que faz parte da família Celastracea que compreende cerca de 88 gêneros. Essa família é composta por aproximadamente 900 espécies de ampla distribuição nas regiões tropicais e subtropicais de todo o mundo (JORGE et al., 2004; SANTOS, 2011).

O gênero Maytenus é formado por espécies arbustivas ou arbóreas encontradas em matas e campos (ESTEVAM et al., 2009) que são largamente utilizadas pelos povos da América do Sul. As plantas desse gênero são ricas em metabólitos bioativos da classe dos terpenos ou isoprenóides (GUTIEERREZ et al., 2007) e atividades biológicas tais como antiinflamatória (SOSA et al., 2007), antiulcerogênica (MOTA et al., 2008), antimalárica (MUREGI et al., 2007), antioxidante (BRUNNI et al., 2006), antinociceptiva (NIERO et al., 2006), antitumoral (SHIROTA et al., 1994) e anticonvulsivante (QUINTANS-JÚNIOR et al., 2008) já foram relatadas para diversas espécies desse gênero.

As espécies do gênero Maytenus são conhecidas e aplicadas pelo conhecimento popular no combate a dores, afecções do trato gastrointestinal e geniturinário. Em associação à aplicação empírica pela população, já existe comprovação científica de sua atividade antifúngica, antioxidante e antimicrobiana, relacionando essas ações à sua composição rica em metabólitos secundários como compostos fenólicos, flavonoides, terpenos e taninos (SANTOS; SANTOS, 2017).

Sendo assim, propõe-se o estudo laboratorial da capacidade de captura do radical DPPH pelo extrato etanólico da casca da Maytenus rigida Mart., estudo de seu conteúdo de metabólitos secundários através triagem fitoquímica.

\section{Procedimento metodológico}




\section{Material vegetal e extratos}

Para o preparo do extrato etanólico, a casca da espécie Maytenus rigida foi colhida no município de Arapiraca, moída e submetida a processo de secagem. O material resultante desses processos foi pesado e adicionado de álcool etílico. A etapa seguinte consistiu em diluições consecutivas e rotaevaporação, resultando na produção do extrato etanólico puro.

\section{Prospecção fitoquímica}

O extrato da planta utilizada neste trabalho foi submetido à prospecção fitoquímica, seguindo-se a descrição de Matos (1997). Os métodos utilizados nesta abordagem são apenas qualitativos, e a presença de um constituinte pode mascarar a cor indicativa do outro. Esta metodologia teve como objetivo detectar a ocorrência de diversos constituintes químicos presentes na casca da $M$. rigida, onde para os testes sete porções de 3-4 mL de cada amostra, dissolvidos em etanol, foram colocados em tubos de ensaio numerados de 1 a 7 .

\section{Avaliação da atividade antioxidante}

A atividade antioxidante foi determinada experimentalmente pelo método DPPH. Para isso, as amostras do extrato etanólico foram diluídas em 4 diferentes concentrações.

Após a produção das diluições, procedeu-se com a transferência de $2,5 \mathrm{ml}$ de cada uma das concentrações para vidros âmbar e a adição de $1 \mathrm{~mL}$ da solução de $\mathrm{DPPH}$, produzida com 0,003g de DPPH e 25,360mL de etanol.

Ainda foram ainda produzidos brancos para cada uma das concentrações, também em triplicata, contendo apenas $2,5 \mathrm{ml}$ das concentrações do extrato.

Em seguida, os vidros âmbar foram colocados em local abrigado da luz onde foram deixadas por trinta minutos, ao final dos quais foram submetidas à leitura das absorbâncias por espectrofotometria.

Como controle negativo foi utilizada uma alíquota de $1 \mathrm{~mL}$ de solução etanólica de DPPH adicionada de $2,5 \mathrm{~mL}$ de etanol.

Para avaliar a ação de captura de radicais, a porcentagem de inibição foi determinada pela seguinte fórmula: $\mathrm{AAO} \%=100-\{[\mathrm{AbsA}-\mathrm{AbsB} \vdash-]-\dashv \mathrm{X} 100\} / \mathrm{AbsC}$ em que Abs A se refere à absorbância da amostra, $\mathrm{Abs} \mathrm{B}$ absorbância de branco e $\mathrm{Abs} \mathrm{C}$ à do controle.

Os resultados da absorvância coletados por espectrofotometria foram agrupados em planilha do programa "Excel for Windows". A partir desses dados foi construída a equação da reta e calculado o CE50 que representa a concentração em que a amostra tem a capacidade de reduzir em 50\% o DPPH. Na determinação do CE50 foi utilizada a fórmula $y=a x+b$, sendo $y=$ 50 e $\mathrm{x}=\mathrm{CE} 50$.

\section{Resultados e discussão}

No que diz respeito à prospecção fitoquímica, pode-se perceber que o extrato analisado tem como componentes: flavonoides, xantonas, chalconas, auronas, flavononois, catequinas, flavononas, xantonas, esteroides livres e saponinas (Tabela 1). Resultados semelhantes já foram observados para outras espécies desse gênero, como é o caso da Maytenus ilicifolia, conhecida popularmente como Espinheira-Santa, que também é comumente aplicada de maneira empírica 
sob a forma de infusões, principalmente no tratamento de gastrite e outros distúrbios do trato gastrointestinal (DE FREITAS et al., 2017).

A presença desses metabólitos secundários é um indício de que as atividades antiinflamatória, analgésica e antimicrobiana da $M$. rígida citadas pela experiência popular realmente podem ser consequência de sua composição rica em metabólitos secundários, que agem na redução de processos patológicos mediados pelo estresse oxidativo e por reações inflamatórias (DOS SANTOS; RODRIGUES, 2017).

Tabela 1: Prospecção fitoquímica do extrato etanólico de Maytenus rígida Mart.

\begin{tabular}{cc}
\hline Constituintes Químicos & Presença ou ausência \\
\hline Fenóis & - \\
Taninos flobatênicos & - \\
Antocianina e antocianidina & + \\
Flavonas, flavonóis e xantonas & + \\
Chalconas e auronas & + \\
Flavononóis & + \\
Catequinas & + \\
Flavononas & + \\
Esteróides & + \\
Saponinas &
\end{tabular}

Fonte: Dados do pesquisador.

$\mathrm{Na}$ avaliação da atividade antiradicalar pelo método de captura do radical orgânico, o extrato etanólico da $M$. rigida apresentou atividade nas concentrações testadas, indicando a presença de metabólitos secundários que podem ser utilizados como substâncias ativas em processos que envolvam a redução do estresse oxidativo como ocorre no tratamento de doenças inflamatórias e neoplásicas (Figura 1). Através dessa metodologia pode ser determinado, ainda, o CE50 do extrato que indica a concentração mínima necessária para a redução de $50 \%$ do radical livre. No caso da espécie estudada esse valor foi de 22,886. Em comparação com outras espécies vegetais utilizadas com frequência pela população brasileira, o extrato estudado se mostrou superior já que a concentração necessária para a redução efetiva de $50 \%$ dos radicais livres é menor, como é o caso da $T$. brasiliensis $($ CE50 $=27,59)$ e $C$. prunifera $($ CE50 $=111,14)$ (SOUSA et al., 2007).

O EE da casca da $M$. rigida também apresentou potencial antioxidante demonstrado pelo método de DPPH. A capacidade antioxidante de vários metabótitos secundários confere um potencial terapêutico com atividades antinociceptiva e antiinflamatória (LI et al., 2002). Tem sido demonstrado que os flavonóides e taninos são capazes de inibir as isoformas induzíveis das enzimas COX e/ou NOS, bem como outros mediadores do processo inflamatório (DE CARVALHO-OKANO, 2005). Portanto, pode sugerir que o EE da casca da M. rigida pode estar agindo, ao menos em parte, devido à presença destes metabólitos e a sua ação antioxidante. 
Revista Ambientale

Revista da Universidade Estadual de Alagoas/UNEAL e-ISSN 2318-454X, Ano 12, Vol. 12 (4), outubro/dezembro (2020)

Figura 1: Atividade antioxidante da espécie $M$. rígida

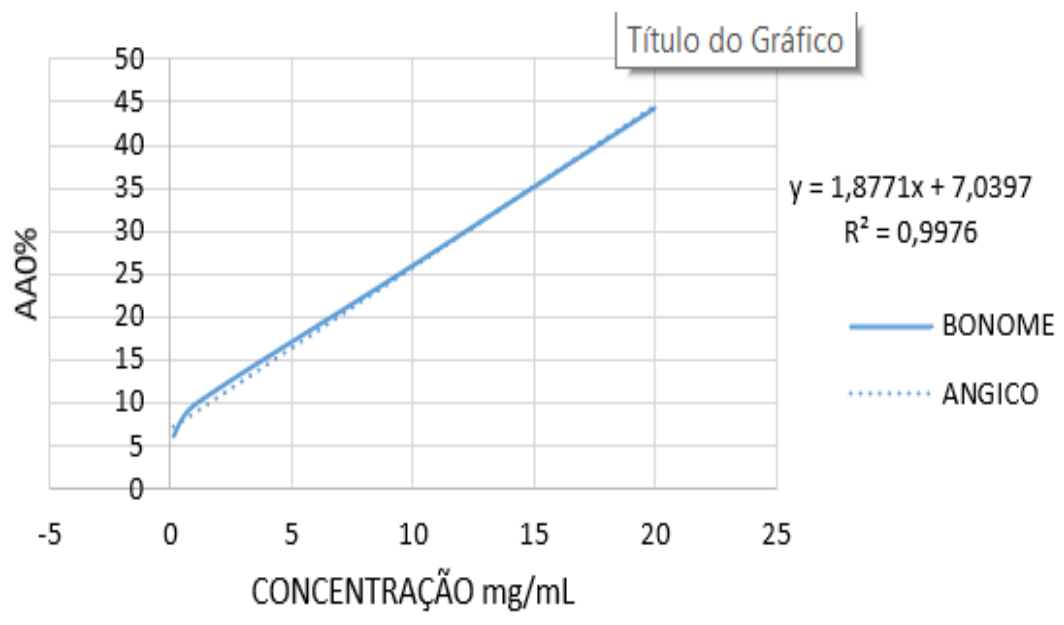

Fonte: Dados do pesquisador, 2019.

\section{Conclusão}

Tendo em vista os resultados apresentados neste trabalho, cujo objetivo central foi avaliar a capacidade antioxidante da casca da $M$. rigida, pode-se considerar que as atividades biológicas (antinociceptiva e anti-inflamatória) estão relacionadas, ao menos em parte, a sua atividade antioxidante. Tais resultados possibilitam reais condições para que esta planta possa ser uma fonte abundante de compostos ativos, cuja pesquisa aprofundada poderá levar à descoberta de uma nova e melhor alternativa terapêutica aos estados patológicos com quadro inflamatório agudo, visto que os flavonóides e taninos são capazes de inibir as isoformas induzíveis das enzimas COX e/ou NOS, bem como outros mediadores do processo inflamatório.

\section{Conflito de interesses}

Os autores deste manuscrito não declararam conflitos de interesse.

\section{Referências bibliográficas}

BRUNI, R. et al. Antimutagenic, antioxidant and antimicrobial properties of Maytenus krukovii bark. Fitoterapia, v. 77, n. 7-8, p. 538-545, 2006.

DE CARVALHO-OKANO, R. M.. Maytenus littoralis Carvalho-Okano (Celastraceae), uma nova espécie para o Brasil. 2005. 
Revista Ambientale

Revista da Universidade Estadual de Alagoas/UNEAL

e-ISSN 2318-454X, Ano 12, Vol. 12 (4), outubro/dezembro (2020)

DE FREITAS, Jamiliana Brito et al. MAYTENUS ILICIFOLIA E SUA AÇÃO GASTROPROTETORA: REVISÃO DE LITERATURA. Mostra Científica da Farmácia, v. 4, n. 2, 2018.

DOS SANTOS, D. S.; RODRIGUES, M. M. F. Atividades farmacológicas dos flavonoides: um estudo de revisão. Estação Científica (UNIFAP), v. 7, n. 3, p. 29-35, 2017.

ESTEVAM, C. S. et al. Perfil fitoquímico e ensaio microbiológico dos extratos da entrecasca de Maytenus rigida Mart. (Celastraceae). Rev. bras. farmacogn., João Pessoa, v. 19, n. 1b, p. 299-303, Mar. 2009.

GUTIÉRREZ, F. et al. Terpenoids from the medicinal plant Maytenus ilicifolia. Journal of natural products, v. 70, n. 6, p. 1049-1052, 2007.

JORGE, R.M. et al. Evaluation of antinociceptive, antiinflammatory and antiulcerogenic activities of Maytenus ilicifolia. Journal of the Ethnopharmacology, v.94, p.93- 100, 2004.

MOTA, K. S. L. et al. Evaluation of the toxicity and antiulcerogenic activity of the ethanol extract of Maytenus obtusifolia Mart. Leaves. Revista Brasileira de Farmacognosia, v. 18, n. 3, p. 441-446, 2008.

MUREGI, F. W. et al. Antimalarial activity of methanolic extracts from plants used in Kenyan ethnomedicine and their interactions with chloroquine (CQ) against a CQ-tolerant rodent parasite, in mice. Journal of Ethnopharmacology, v. 111, n. 1, p. 190-195, 2007.

NIERO, Rivaldo et al. A new triterpene with antinociceptive activity from Maytenus robusta. Natural Product Research, v. 20, n. 14, p. 1315-1320, 2006.

QUINTANS JÚNIOR, L. J. et al. Plants with anticonvulsant properties: a review. Revista Brasileira de Farmacognosia, v. 18, p. 798-819, 2008.

SANTOS, L. C.. Avaliação da atividade biológica da fração hexânica da entrecasca de Maytenus rígida Mart. 2017.

SANTOS, V. L. et al. Avaliação da atividade antimicrobiana de Maytenus rigida Mart. (Celastraceae). Revista Brasileira de Plantas Medicinais, v. 13, n. 1, p. 68-72, 2011.

SANTOS, V. L. et al. Pharmacological studies of ethanolic extracts of Maytenus rigida Mart (Celastraceae) in animal models. Rev. bras. farmacogn., João Pessoa, v. 17, n. 3, p. 336-342, Sept. 2007.

SILVA, G. D. F. et al. Antioxidant activity of Maytenus imbricata Mart., Celastraceae.Rev. bras. farmacogn., João Pessoa, v. 19, n. 2b, p. 530-536, June 2009.

SOSA, S. et al. Anti-inflammatory activity of Maytenus senegalensis root extracts and of maytenoic acid. Phytomedicine, v. 14, n. 2-3, p. 109-114, 2007. 
Revista Ambientale

Revista da Universidade Estadual de Alagoas/UNEAL

e-ISSN 2318-454X, Ano 12, Vol. 12 (4), outubro/dezembro (2020)

SOUSA, C. M. de M. et al. Fenóis totais e atividade antioxidante de cinco plantas medicinais. Química nova, v. 30, n. 2, p. 351-355, 2007. 